\title{
Retention models: "tis the gift to be simple
}

\author{
Nils P. Johnson, MD, MS, and K. Lance Gould, $M^{a}{ }^{a}$ \\ a Weatherhead PET Center, Division of Cardiology, Department of Medicine, McGovern Medical \\ School at UTHealth and Memorial Hermann Hospital, Houston, TX
}

Received Sep 24, 2021; accepted Sep 24, 2021

doi: $10.1007 / \mathrm{s} 12350-021-02827-z$

\section{See related article, pp. 2583-2594}

Beginning almost 40 years ago, the senior author of this editorial (KLG) and colleagues published two papers outlining the theory ${ }^{1}$ and practical implementation $^{2}$ of a so-called "simplified" (part of one paper's ${ }^{2}$ title) approach to quantifying myocardial perfusion using positron emission tomography (PET). As noted 25 years ago, this approach offers advantages over timeactivity curve (TAC) fitting "due to complexity of flow models and poor quality of the brief serial images used for flow determinations.", 2 The recent publication by van Tosh et al. ${ }^{3}$ comparing a 1-tissue compartment model using TAC against a retention model (the more formal term for our simplified approach) provides an opportunity to revisit the issue. Are "simplified", methods no longer necessary with modern PET cameras and more powerful computing? Or does a retention model continue to provide enduring and objective advantages?

\section{REGIONAL AND PRACTICAL}

Let us begin with what we want clinically when quantifying myocardial perfusion and then work backwards to examine design choices. Ultimately quantitative perfusion allows for two major decisions. First, the size and severity of near transmural perfusion defects indicates or refutes the physiologic necessity of revascularization, ${ }^{4}$ while their spatial pattern suggests general anatomic targets. Second, stress-induced

Reprint requests: Nils P. Johnson, MD, MS, Weatherhead PET Center, Division of Cardiology, Department of Medicine, McGovern Medical School at UTHealth and Memorial Hermann Hospital, Houston, TX; Nils.Johnson@uth.tmc.edu

J Nucl Cardiol 2022;29:2595-8.

1071-3581/\$34.00

Copyright (C) 2021 American Society of Nuclear Cardiology. subendocardial perfusion defects represent a common physiologic substrate for angina, electrocardiographic changes, and myocardial injury; they provide a mechanistic explanation for the patient and referring physician, but generally require only medical treatment. ${ }^{5}$

Already these clinical requirements make clear the necessity of regional quantification. While global perfusion correlates with risk factors, atherosclerotic burden, and clinical outcomes, revascularization provides vessel-level therapy. Global flow capacity can remain intact despite significant regional epicardial disease that requires intervention. ${ }^{5}$ As written nearly four decades ago, it "does not permit measurement of regional differences in extraction but instead provides only an average extraction fraction for the whole organ, various parts of which may have widely divergent extractions.", 1

In addition to providing the smallest possible regional flow values, quantifying myocardial perfusion must remain practical. At our own center we have done so for every single stress cardiac PET study since April 2007. As such, imaging and processing protocols should be straightforward yet robust against mild patient motion (whether gradual or abrupt), shifting abdominal contents while supine, respiratory variation (even during mild tachypnea often induced by vasodilator stress), and cardiac motion regardless of the rhythm. Any technique can look great with meticulous processing and the ideal patient. But every study? Every day? For almost 15 years?

\section{RETENTION}

Table 1 contrasts tradeoffs between retention models and TAC fitting, here explored in more detail. At its most basic, a retention model corrects for the roll-off seen with perfusion tracers like Rb-82 and N-13 ammonia. While sometimes described as a crippling flaw, two factors make this non-linear relationship less important clinically as explained visually in Figure 1. First, flow models correct for differential retention, "bending', the 
Table 1. Trade-offs between quantitative myocardial flow models

\begin{tabular}{lll}
\hline Property & Retention model & Time-activity curve model \\
\hline Complexity of acquisition and processing & Lower & Higher \\
Customized location of arterial input & Straightforward & Not routine \\
Arterial input noise & Lower & Higher \\
Sensitivity to motion during acquisition & Lower & Higher \\
Partial volume corrections & Explicit & Hidden \\
Adjustment for tracer timing and bolus shape & Not possible & Mandatory \\
Sophistication of tracer kinetics & Modest & Unlimited \\
\hline
\end{tabular}

curves back to a straight line. Second, flows above 1 $\mathrm{cm}^{3} \cdot \mathrm{min}^{-1} \cdot \mathrm{g}^{-1}$ and certainly above $2 \mathrm{~cm}^{3} \cdot \mathrm{min}^{-1} \cdot \mathrm{g}^{-1}$ do not indicate severe atherosclerosis in need of revascularization. Therefore, "shades of grey" among high flows impart at best modest clinical impact since wellestablished algorithms exist for treating risk factors or coronary disease medically independent of absolute flow assessment.

Retention conceptually equals the ratio of myocardial uptake to arterial input. Because a retention model assumes constant myocardial uptake after extraction, only a single "late" image must be acquired, typically starting 2 minutes after Rb-82 injection or 80 seconds after N-13 ammonia injection (shorter due to recirculation of its non-extractable metabolites like glutamine). This image offers high signal-to-noise ratio due to its long duration (5 minutes for $\mathrm{Rb}-82$ and 10 minutes for $\mathrm{N}-13$ ammonia, although the vast majority of counts accumulate in the first half of this period). Additionally, acquiring a single image over several minutes tolerates

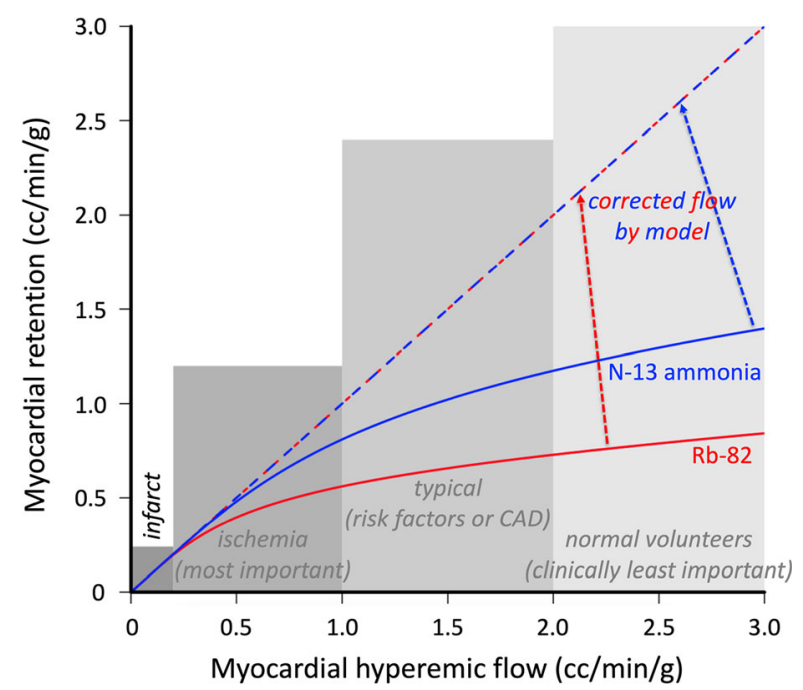

Figure 1. Myocardial retention of various radiotracers with increasing flow. mild motion and so-called "creep" artifacts that can plague fixed regions of interest (ROI) on serial images of short duration. While an ROI can be adjusted frame-toframe and motion correction algorithms applied, a single myocardial uptake image naturally incorporates resilience. As a consequence of the high count density in this late image, spatial confidence increases and permits regional myocardial uptake quantification per pixel for accurate and artery-specific size/severity of abnormalities. TAC per pixel are so statistically noisy as to require average curves within arbitrary, large regions of interest in assumed arterial distributions that often overlap multiple different arteries even for the 17-segment model.

Arterial input sums the "area under the curve" near the origin of the coronary arteries. Typical structures examined for this purpose include the left atrium, ascending aorta, or left ventricular blood pool. However, the optimal location in each scan must be customized given many factors that preclude cookie-cutter selection. ${ }^{6}$ This issue is not unique to cardiac PET, having been observed with cardiac magnetic resonance perfusion imaging too. ${ }^{7}$ As such, TAC models could also optimize their arterial input location since this feature is not strictly part of the retention model. However, obtaining a single "early" image of sizable duration ( 2 minutes for $\mathrm{Rb}-82$ and 80 seconds for $\mathrm{N}-13$ ammonia) makes the issue visually clear and reduces noise for the retention input, unlike noisy TAC whose comparisons among locations requires greater effort and uncertainty. Finally, because only the area under the input curve matters, not its specific shape, a retention model carries inbuilt protection against unimportant changes in tracer delivery trajectory among generators or technicians.

Retention thus equals myocardial activity in a certain region (units like $\mu \mathrm{Ci} \cdot \mathrm{cm}^{-3}$ ) divided by the arterial input in a certain region (units like $\mu \mathrm{Ci} \cdot \mathrm{cm}^{-3}$ multiplied by the duration of the early image for final units like $\mu \mathrm{Ci} \cdot \mathrm{cm}^{-3} \cdot \mathrm{min}$ ) then divided by the myocardial 
density (taken to be $1.05 \mathrm{~g} \cdot \mathrm{cm}^{-3}$ ). Hence the units work out as expected: $\left(\mu \mathrm{Ci} \cdot \mathrm{cm}^{-3}\right) /\left(\mu \mathrm{Ci} \cdot \mathrm{cm}^{-3} \cdot \min \right) /\left(\mathrm{g} \cdot \mathrm{cm}^{-3}\right)=$ $\mathrm{cm}^{3} \cdot \mathrm{min}^{-1} \cdot \mathrm{g}^{-1}$. This retention can be converted to flow using the curves in Figure 1 that have been explicitly determined by several groups for $\mathrm{Rb}-82$ and $\mathrm{N}-13$ ammonia. $^{8}$

Two final factors modify the retention value, namely the partial volume (PV) effects of imaging the myocardial activity and arterial input. Actually, these coefficients represent the net balance of reduced activity from PV loss and increased activity from spillover due to adjacent structures (for example, hot blood pool spillover into the myocardial ROI). Due to the organization of the early and late images (arterial and myocardial uptake dominate their respective acquisitions), spillover effects can be neglected. Generally, the arterial input requires no PV correction on modern scanners given the large size of these structures (aorta and left atrium). Partial volume correction of myocardial uptake can pose an almost intractable problem for TAC as well as retention models since it varies during the cardiac cycle ${ }^{9}$ (larger in diastole, smaller in systole), potentially over the left ventricle (due to differential wall thickness from hypertrophy, wall motion abnormalities, and thinning from prior infarction), and among patients due to the interplay between the orientation of the cardiac axis and PET scanner axis (with anisotropy from in-plane vs between-plane resolution). Retention models require a fixed PV factor as opposed to TAC fitting that generally allows it to vary. We explicitly derive a PV correction from phantoms on each of our scanners, namely myocardial activity increases by $1 /$ 0.90 or $11 \%$ when computing the retention with $\mathrm{Rb}-82$ on our GE Discovery ST with its associated reconstruction parameters and filters. ${ }^{9}$ To our knowledge, no software package displays the resulting PV value from TAC models. Consequently, PV corrections from TAC fitting cannot be examined by the physician to ensure that these values remain physiologic.

\section{CURVE FITTING VS RETENTION}

With this background in retention versus TAC models, we can approach the new results from van Tosh et $a l{ }^{3}$ They retrospectively analyzed rest and stress $\mathrm{Rb}$ 82 cardiac PET images from 123 subjects for a total of 246 scans. Each scan was analyzed for absolute perfusion using two techniques, namely a retention model and TAC fitting for a 1-tissue compartment model. The retention modeling software was Corridor4DM, although no name was provided for the TAC fitting software (likely the Emory Cardiac Toolbox as three coauthors disclosed royalties from Syntermed and this program is listed as an abbreviation on the first page, without actually appearing in the text of the manuscript).

An automated algorithm rated image quality in seven categories: inconsistent frame duration (never occurred in this cohort), scanner saturation (occurred twice at stress, never at rest for this 3-dimensional GE D710 scanner), inability to detect blood curve peak (occurred once at stress, never at rest), inappropriate blood peak width (occurred once at rest and once at stress), flat blood curve tail (never occurred), gradual patient motion (occurred once at stress, never at rest), and abrupt patient motion (occurred twice at rest and three times at stress). Thus, we can appreciate that stress imaging poses greater challenges for quality control ( 8 affected stress scans vs 3 rest scans) and that motion represents the major mechanism (6 of 11 failed quality checks).

Despite only 9 of 123 subjects with any quality issue, their absolute stress perfusion and flow reserve using the TAC model decreased significantly compared to subjects with good quality (stress 0.93 vs 1.54 $\mathrm{cm}^{3} \cdot \mathrm{min}^{-1} \cdot \mathrm{g}^{-1}, P=0.03$; reserve 1.45 vs $2.05, P=$ $0.01)$. Conversely perfusion and flow reserve using a retention model were higher but did not reach statistical significance. While limited by small numbers of scans with quality problems, this result emphases the robustness of the retention model. In this study both models appear to have used a fixed arterial input ROI (introducing noise versus an optimized ROI for each scan) and either did not report PV corrections (retention model) or reported values that seem extreme for modern scanners (0.50 at rest and 0.43 at stress for the myocardium, indicating that half of myocardial counts are lost with imaging, in contrast to phantom data suggesting a value closer to 0.9 , i.e. only $10 \% \operatorname{loss}^{8}$ ).

Future work building on this result might explore two alternative reference standards instead of simply comparing paired flow values using a Bland-Altman approach (although their use of a logarithmic transformation for such an analysis is not standard and tends to blunt differences). First, low stress perfusion and CFR values can cause frank ischemia during imaging (stressinduced defect with severe angina and/or significant STsegment depression). Do values from a retention model or TAC fitting better predict such endpoints? Second, patient outcomes as modified by conservative therapy vs revascularization provide a meaningful clinical yardstick. Does one model outperform the other in this regard? Finally, sequential, repeated, test/retest quantitative measurements in the same patient provide a coefficient of variation that we might expect to be larger for the TAC than the retention model for the above reasons. 


\section{CONCLUSION}

As summarized in Table 1, cardiac PET perfusion models offer tradeoffs with no universal champion-as with many choices in life. However, the important work by van Tosh et $a l^{3}$ lends further support to the robustness of retention models. Prior literature from groups other than our own has encouragingly noted that:

- "when we restricted correlation analysis to the physiological flow range $<3 \mathrm{~mL} \cdot \mathrm{g}^{-1} \cdot \mathrm{min}^{-1}$, which covers most of the values observed in the clinical setting, the retention approach yielded a better agreement with microsphere flow. This is most likely explained by the intrinsic noise and variability of dynamic Rb-82 images, which translate into noise of TACs and thus inaccuracies of curve fitting especially when flow, and thus the amount of delivered radiotracer and resultant imaging signal, are low. The retention approach is more robust in this regard because only an activity concentration determined from a longer time interval and an integral under the arterial blood curve go into the equation. Our data thus suggest that the retention approach is a preferable method for clinical quantification of flow in humans, where very high flow is unlikely and data noise is expected to be larger than in our animal model.",10

- "retention model may have higher sensitivity for detection and localization of abnormal flow ... retention approach yielded a better agreement with microsphere flow ... most likely explained by the intrinsic noise and variability of ... time-activity curves ... retention approach is more robust ... preferable method for clinical quantification., 8

While retention models were initially self-described and externally regarded as "simplistic", the quote in the title from the classic Shaker song reminds us that simplicity can be its own gift.

\section{Disclosure}

NPJ and KLG received internal funding from the Weatherhead PET Center for Preventing and Reversing Atherosclerosis; and have pending patents on diagnostic methods for quantifying aortic stenosis and TAVI physiology, and on algorithms to correct pressure tracings from fluid-filled catheters. NPJ has received significant institutional research support from St. Jude Medical (CONTRAST, NCT02184117) and Philips/Volcano Corporation (DEFINE-FLOW, NCT02328820) for other studies using intracoronary pressure and flow sensors; and has an institutional licensing agreement with Boston Scientific for the smart minimum FFR algorithm commercialized under 510(k) K191008. KLG is the 510(k) applicant for CFR Quant (K113754) and HeartSee (K143664, K171303, and K202679), software packages for cardiac PET image processing, analysis, and absolute flow quantification.

\section{References}

1. Mullani NA, Goldstein RA, Gould KL, Marani SK, Fisher DJ, O'Brien HA Jr, et al. Myocardial perfusion with rubidium-82. I. Measurement of extraction fraction and flow with external detectors. J Nucl Med 1983;24:898-906.

2. Yoshida K, Mullani N, Gould KL. Coronary flow and flow reserve by PET simplified for clinical applications using rubidium- 82 or nitrogen-13-ammonia. J Nucl Med 1996;37:1701-12.

3. Van Tosh A, Cao JJ, Votaw JR, Cooke CD, Palestro CJ, Nichols KJ. Clinical implications of compromised ${ }^{82} \mathrm{Rb}$ PET data acquisition. J Nucl Cardiol 2021. https://doi.org/10.1007/s12350-02102774-9.

4. Gould KL, Kitkungvan D, Johnson NP, Nguyen T, Kirkeeide R, Bui L. Mortality prediction by quantitative PET perfusion expressed as coronary flow capacity with and without revascularization. JACC Cardiovasc Imaging 2021;14:1020-34.

5. Gould KL, Johnson NP. Coronary physiology beyond coronary flow reserve in microvascular angina: JACC state-of-the-art review. J Am Coll Cardiol 2018;72:2642-62.

6. Vasquez AF, Johnson NP, Gould KL. Variation in quantitative myocardial perfusion due to arterial input selection. JACC Cardiovasc Imaging 2013;6:559-68.

7. Franks R, Milidonis X, Schneider T, Sánchez-González J, Plein S, Chiribiri A. Impact of the arterial input sampling location on CMR first-pass myocardial perfusion quantification. JACC Cardiovasc Imaging 2020;13:2693-5.

8. Renaud JM, DaSilva JN, Beanlands RS, DeKemp RA. Characterizing the normal range of myocardial blood flow with ${ }^{82}$ rubidium and ${ }^{13} \mathrm{~N}$-ammonia PET imaging. J Nucl Cardiol 2013;20:578-91.

9. Johnson NP, Sdringola S, Gould KL. Partial volume correction incorporating $\mathrm{Rb}-82$ positron range for quantitative myocardial perfusion PET based on systolic-diastolic activity ratios and phantom measurements. J Nucl Cardiol 2011;18:247-58.

10. Lautamäki R, George RT, Kitagawa K, Higuchi T, Merrill J, Voicu C, et al. Rubidium-82 PET-CT for quantitative assessment of myocardial blood flow: Validation in a canine model of coronary artery stenosis. Eur J Nucl Med Mol Imaging 2009;36:57686.

Publisher's Note Springer Nature remains neutral with regard to jurisdictional claims in published maps and institutional affiliations. 